\title{
APPENDIX D: SAMPLE POST-VISIT PATIENT SURVEY
}

1. Were you satisfied with today's care?

2. How did you find the staff treated you?

3. Did the provider make good eye contact with you?

4. Did you feel that your needs were met?

5. Did the software (Internet site, application, etc.) work as it was supposed to?

6. Would you recommend this service to friends and family?

These questions can be made to be scaled upon 1-10, yes or no, circle an appropriate answer, or any other method to collect information. 